\title{
A Probabilistic Damage Identification Approach for Structures under Unknown Excitation and with Measurement Uncertainties
}

\author{
Ying Lei, ${ }^{1}$ Ying Su, ${ }^{1}$ and Wenai Shen ${ }^{2}$ \\ ${ }^{1}$ Department of Civil Engineering, Xiamen University, Xiamen 361005, China \\ ${ }^{2}$ Department of Civil and Environmental Engineering, The Hong Kong Polytechnic University, Hung Hom, Kowloon, Hong Kong
}

Correspondence should be addressed to Ying Lei; ylei@xmu.edu.cn

Received 26 December 2012; Revised 4 May 2013; Accepted 26 May 2013

Academic Editor: Xiaojun Wang

Copyright (C) 2013 Ying Lei et al. This is an open access article distributed under the Creative Commons Attribution License, which permits unrestricted use, distribution, and reproduction in any medium, provided the original work is properly cited.

Recently, an innovative algorithm has been proposed by the authors for the identification of structural damage under unknown external excitations. However, identification accuracy of this proposed deterministic algorithm decreases under high level of measurement noise. A probabilistic approach is therefore proposed in this paper for damage identification considering measurement noise uncertainties. Based on the former deterministic algorithm, the statistical values of the identified structural parameters are estimated using the statistical theory and a damage index is defined. The probability of identified structural damage is further derived based on the reliability theory. The unknown external excitations to the structure are also identified by statistical evaluation. A numerical example of the identification of structural damage of a multistory shear-type building and its unknown excitation shows that the proposed probabilistic approach can accurately identify structural damage and the unknown excitations using only partial measurements of structural acceleration responses contaminated by intensive measurement noises.

\section{Introduction}

Structural damage detection is an important task for structural health monitoring [1-5]. Usually, it is straightforward to identify structural damage based on tracking the changes of the identified values of structural physical parameters, for example, the degrading of element stiffness parameters. In practice, it is often impossible to deploy so many sensors that accurately measure all excitation inputs and response outputs of systems. It is highly desirable to deploy as few sensors as possible, so it is essential to explore efficient algorithms which can identify structural damage utilizing only a limited number of measured responses of structures subject to some unknown (unmeasured) excitations.

In the past decades, some researchers have proposed algorithms for simultaneous identification of structural parameters and unknown excitation, for example, the iterative leastsquare estimation approach $[6,7]$, the statistical average algorithm [8], the recursive least-square estimation [9], genetic algorithms [10], hybrid identification method [11], the dynamic response sensitivity method [11], the extended Kalman filter with unknown excitation inputs (EKF-UI) [12], the sequential nonlinear least-square estimation (SNLSE) [13], and structural parameters and dynamic loading identification from incomplete measurements [14]. However, these approaches suffered the deficiencies of either all structural response being assumed available or the analytical and numerical identification procedures being rather complex.

Recently, an innovative algorithm has been proposed by the authors for the identification of structural damage under unknown excitations using limited measurements of structural acceleration responses $[15,16]$. The proposed algorithm is based on the sequential utilization of the extended Kalman estimator [17] for the recursive estimation of the extended state vector of a structure and the least-square estimation of its unknown excitation; that is, recursive solution for extended state vector is initially estimated followed by the subsequent estimation of the unknown excitation via leastsquare estimation. Thus, proposed algorithm simplifies the identification problem compared with previous simultaneous identification approaches $[18,19]$. Structural damage is detected from the changes of structural parameters at the element level, such as the degradation of identified element stiffness parameters. Such a straightforward derivation and 
analytical solution are not available in the previous literature. However, former numerical examples indicated that the identification accuracy of this proposed deterministic algorithm decreased with the increase of the measurement noise level $[15,16]$. Therefore, it is necessary to develop an approach which can avoid the false identification of damages in the deterministic identification algorithm induced by the relatively high level of measurement noise.

Since the inevitable measurement noises are intrinsically uncertain, the identification of structural parameter and external excitation using measurements with intensive measurement noises is essentially an uncertain problem [20, 21]. The identification performed by deterministic methods often leads to incorrect identification results of structural damages and a disagreement between the identified unknown excitation and its true value while consideration of uncertainties has received more and more attention in recent years [2226]. In this paper, a probabilistic approach is proposed for the identification of structural damage under unknown external excitations and with measurement noise uncertainties. Based on the deterministic algorithm, the statistical values of the identified structural parameters are estimated, and the probability of identified structural damage is further derived using the statistical theory and probability method. The rest of the paper is organized as follows. Section 2 briefly introduces the former deterministic algorithm for the identification of structural damage under unknown external excitations, Section 3 presents the proposed probabilistic identification approach based on the improvement of the deterministic algorithm using the statistical and probability theory, Section 4 shows a numerical example of the identification of structural damage of a multistory shear-type frame building and its unknown excitation to demonstrate the proposed probabilistic approach, and Section 5 gives the conclusions of the paper.

\section{Brief Introduction of the Deterministic Algorithm for Identification of Structural Damage under Unknown Excitations}

The equations of motion of a linear structural system subject to unknown external excitation can be written as

$$
\mathbf{M} \ddot{\mathbf{x}}(t)+\mathbf{C} \dot{\mathbf{x}}(t)+\mathbf{K x}(t)=\mathbf{B}^{u} \mathbf{f}^{u}(t),
$$

in which $\mathbf{x}(t), \dot{\mathbf{x}}(t)$, and $\ddot{\mathbf{x}}(t)$ are the vectors of displacement, velocity, and acceleration response, respectively; $\mathbf{M}, \mathbf{C}$, and $\mathbf{K}$ are the mass, damping, and stiffness matrices, respectively; $\mathbf{f}^{u}(t)$ is an unmeasured external excitation vector; and $\mathbf{B}^{u}$ is the influence matrix associated with $\mathbf{f}^{u}(t)$. Usually, the mass of a structural system can be estimated with accuracy based on its geometry and material information.

2.1. Estimation of the Extended State Vector. The extended state vector of the system is defined as

$$
\begin{aligned}
\mathbf{X} & =\left[\mathbf{X}_{1}^{T}, \mathbf{X}_{2}^{T}, \boldsymbol{\theta}^{T}\right]^{T} ; \quad \mathbf{X}_{1}=\mathbf{x} ; \\
\mathbf{X}_{2} & =\dot{\mathbf{x}} ; \quad \boldsymbol{\theta}^{T}=\left[\theta_{1}, \theta_{2}, \ldots, \theta_{n}\right]^{T},
\end{aligned}
$$

where $\boldsymbol{\theta}^{T}$ is a vector of the $n$-unknown structural parameters, such as damping and stiffness parameters. As the structural parameters are constant, (1) can be written in the following general nonlinear differential state equations $[15,16]$ :

$$
\dot{\mathbf{X}}=\mathbf{g}\left(\mathbf{X}, \mathbf{f}^{u}\right)
$$

Usually, only a limited number of accelerometers are deployed in structures to measure acceleration responses. Therefore, the discretized observation equation can be expressed as

$$
\mathbf{y}[k]=\mathbf{h}(\mathbf{X}[k])+\mathbf{G}^{u} \mathbf{f}^{u}[k]+\mathbf{v}[k],
$$

where $\mathbf{G}^{u}=\mathbf{D M}^{-1} \mathbf{B}^{u} ; \mathbf{h}(\mathbf{X}[k])=\mathbf{D M}^{-1}\left\{-\left(\mathbf{C}_{\boldsymbol{\theta}}\right) \mathbf{X}_{2}[k]-(\mathbf{K})_{\boldsymbol{\theta}}\right.$ $\left.\mathbf{X}_{1}[k]\right\}$ in which $\mathbf{y}[k]$ is observation vector (measured acceleration responses) at time $t=k \times \Delta t$ with $\Delta t$ being the sampling time step, $(\mathbf{C})_{\boldsymbol{\theta}}$ represents elements in the damping matrix $\mathbf{C}$ composed by the unknown parameters of damping in the parametric vector $\boldsymbol{\theta},(\mathbf{K})_{\boldsymbol{\theta}}$ represents the constitution of stiffness matrix $\mathbf{K}$ analogously, $\mathbf{f}^{u}[k], \mathbf{X}_{1}[k]$ and $\mathbf{X}_{2}[k]$ are the corresponding discretized values at time $t=k \times \Delta t, \mathbf{D}$ is the matrix associated with the locations of accelerometers, and $\mathbf{v}[k]$ is the measured noise vector assumed to be a Gaussian white noise vector with zero mean and a covariance matrix $\mathbf{E}\left[\mathbf{v}_{i} \mathbf{v}_{j}^{T}\right]=\mathbf{R}_{i j} \delta_{i j}$, where $\delta_{\mathrm{ij}}$ is the Kronecker delta.

Based on the extended Kalman estimator $[15,16]$, the extended state vector at time $t=(k+1) \times \Delta t$ can be estimated with the observation of $(\mathbf{y}[1], \mathbf{y}[2], \ldots, \mathbf{y}[k])$ as follows:

$$
\begin{gathered}
\widehat{\mathbf{X}}[k+1 \mid k]=\widetilde{\mathbf{X}}[k+1 \mid k]+\mathbf{K}[k] \\
\times\{\mathbf{y}[k]-h(\widehat{\mathbf{X}}[k \mid k-1], \mathbf{f}[k]) \\
\left.-\mathbf{G}^{u} \widehat{\mathbf{f}}^{u}[k \mid k]\right\},
\end{gathered}
$$

in which

$$
\widetilde{\mathbf{X}}[k+1 \mid k]=\widehat{\mathbf{X}}[k \mid k-1]+\int_{t[k]}^{t[k+1]} \mathbf{g}\left(\mathbf{X}, \mathbf{f}^{u}\right) d t,
$$

where $\widehat{\mathbf{X}}[k+1 \mid k]$ and $\widehat{\mathbf{f}}^{u}[k \mid k]$ are the estimation of $\mathbf{X}[k+1]$ and $\mathbf{f}^{u}[k]$ given $(\mathbf{y}[1], \mathbf{y}[2], \ldots, \mathbf{y}[k])$, respectively, and $\mathbf{K}[k]$ is the Kalman gain matrix $[15,16]$.

However, since the external excitation $\mathbf{f}^{u}(t)$ is unknown, it is impossible to obtain the recursive solution for the extended state vector by the classical extended Kalman estimator alone.

2.2. Identification of the Unknown Excitations. Under the conditions: (i) the number of output measurements is greater than that of the unknown excitations and (ii) measurements (sensors) are available at all DOFs where the unknown excitation $\mathbf{f}^{u}(t)$ acts; that is, matrix $\mathbf{G}^{u}$ in (4) is nonzero; the unknown excitations at time $t=(k+1) \times \Delta t$ can be estimated from (4) by the least-square estimation as $[15,16]$

$$
\begin{aligned}
\widehat{\mathbf{f}}^{u}[k+1 \mid k+1]= & {\left[\left(\mathbf{G}^{u}\right)^{T} \mathbf{G}^{u}\right]^{-1}\left(\mathbf{G}^{u}\right)^{T} } \\
& \times\{\mathbf{y}[k+1]-\mathbf{h}(\widehat{\mathbf{X}}[k+1 \mid k])\},
\end{aligned}
$$


in which $\hat{\mathbf{f}}^{u}[k+1 \mid k+1]$ is the estimation of $\mathbf{f}^{u}[k+1]$ given the observation of $(\mathbf{y}[1], y[2], \ldots, y[k+1])$.

Therefore, the proposed algorithm can identify structural parameters and unknown excitation in a sequential manner, which simplifies the identification problem compared with other simultaneous identification work. Structural damage is detected from the changes of structural parameters at the element level, such as the degradation of identified element stiffness parameters. Such a straightforward derivation and analytical solution are not available in the previous literature $[15,16]$.

However, former numerical examples indicated that the identification accuracy of this proposed deterministic algorithm decreases under high level of measurements noise [15, 16]. The identification performed by using the deterministic algorithm leads to incorrect identification results of structural damages and a disagreement between the identified excitation and its true value. Consequently, it is necessary to develop an approach for identifying the structural damage and unknown excitation when the measurements are contaminated by intensive measurement noises.

\section{A Probabilistic Approach for the Identification of Structural Damage with Intensive Measurement Noises}

Since the inevitable measurement noises are intrinsically uncertain, identification of structural parameter and unknown excitation using measurements with intensive measurement noises is essentially an uncertain problem. A probabilistic approach is proposed herein based on the deterministic algorithm described in Section 2.

3.1. The Statistical Results of Identification Values. In the observed equation, (4), the measured noise vector is assumed to be a Gaussian white noise vector; that is, uncertainties in the measured responses are assumed as normally distributed random variables. Then the measured acceleration response vector $\mathbf{y}$ is an observation vector with uncertainties. In practice, many sets of measured accelerations can be obtained by repetitious experiments or long-term measurement of structures. In the numerical simulation, many sets of measured accelerations can be obtained by the theoretically computed responses superimposed with many sets of measurement noise with uncertainties. Then, each set of the measured accelerations is used as an observation vector to identify the structural parameters and unknown excitation by using the deterministic identification algorithm in Section 2. Therefore, many sets of identified results can be obtained. The statistical parameters of the identified parameters can then be estimated by the statistical theory for example, the mean and standard deviations of identified structural element stiffness can be calculated, respectively, by

$$
\mu_{i}=\frac{1}{n} \sum_{j=1}^{n} k_{i j} ; \quad \sigma_{i}=\sqrt{\frac{1}{n-1} \sum_{j=1}^{n}\left(k_{i j}-m_{i}\right)^{2}},
$$

in which $\mu_{\mathrm{i}}$ and $\sigma_{\mathrm{i}}$ are the mean and standard deviations of the $n$-sets of identified stiffness of the $i$ th structural element $k_{i}$, respectively.

Then, a damage index $D_{i}$ for the $i$ th structural element is defined as

$$
D_{i}=\frac{\left(\mu_{i}^{d}-\mu_{i}^{u}\right)}{\mu_{i}^{u}},
$$

in which $\mu_{i}^{d}$ and $\mu_{i}^{u}$ are the mean values of the identified $i$ th structural element stiffness in the damaged and undamaged structure, respectively. Thus, the damage index $D_{i}$ tracks the degrading of the identified $i$ th structural element stiffness and can also reflect its damage severity.

Analogously, the effect of uncertainties on the identified unknown excitation can be decreased by using the statistical average of multisets of identified input time histories, that is,

$$
\overline{\mathbf{f}}^{u}=\frac{1}{n} \sum_{j=1}^{n} \mathbf{f}_{j}^{u}
$$

where $\overline{\mathbf{f}}^{u}$ is the mean value of the $n$-sets that identified unknown excitation time histories and $\mathbf{f}_{j}^{u}$ is the $j$ th set of identified unknown excitation.

\subsection{The Identification Probability of Structural Damage.} Structural damage is assumed as the degrading of the identified $i$ th structural element stiffness; a random variable of the relative change of the identified $i$ th structural element stiffness in the damaged and undamaged structures is introduced as

$$
r_{i}=\frac{\left(k_{i}^{d}-k_{i}^{u}\right)}{m_{i}^{u}}
$$

where $k_{i}^{d}$ and $k_{i}^{u}$ are the identified values of the $i$ th structural element in the damaged and undamaged structures, respectively.

Then, the probability of structural damages in this study is estimated based on the reliability theory; that is, the probability of structural damages of the $i$ th structural element $P_{D_{i}}$ is identified as

$$
P_{D_{i}}=\int_{r_{i} \leq 0} p\left(r_{i}\right) d r_{i},
$$

where $p\left(r_{\mathrm{i}}\right)$ is the probability density function of the random variable $r_{\mathrm{i}}$ in (11).

The random variable $r_{\mathrm{i}}$ can be assumed as a normal random variable. Then, damage probability $P_{D_{i}}$ can be estimated based on the definition of the standard normal distribution as

$$
P_{D_{i}}=1-\Phi\left(\frac{\mu_{r_{i}}}{\sigma_{r_{i}}}\right),
$$

where $\Phi(\bullet)$ denotes the probability of a standard normal distribution. 
Based on the probability $P_{D_{i}}$ defined in (13), the $P_{D_{i}}$ value presents the probability of whether the $i$ th structural element is damaged, and it is in the range of $50 \%-100 \%$. A value of $50 \%$ indicates that the structural element has no damage, whereas a value of larger than $50 \%$ means the occurrence of damage. The closer to $100 \%$ of the $P_{D_{i}}$ value, the larger the damage probability.

\section{A Numerical Simulation Example}

In this paper, a numerical simulation example of the identification of structural damage of a 10-story shear building model and its unknown excitation at the top floor is used to demonstrate the efficiency of the proposed probabilistic approach. The following structural parametric values are used in the numerical study of the 10-story shear building: each story stiffness $k_{1}=k_{2}=\cdots=k_{10}=6.79 \times 10^{3} \mathrm{kN} / \mathrm{m}$, the concentrated mass at each floor level is $m_{1}=3.45 \times$ $10^{3} \mathrm{~kg}, m_{2}=m_{3}=2.65 \times 10^{3} \mathrm{~kg}, m_{4}=m_{5}=\cdots=m_{10}=$ $1.81 \times 10^{3} \mathrm{~kg}$. Rayleigh damping assumption is employed in this study and the two Rayleigh damping coefficients are $\alpha=$ 2.88 and $\beta=5.65$.

The building is excited by a random Gaussian white noise at the top floor; however, this excitation is assumed unknown in the identification process. Partial structural acceleration responses at the 1st, $2 \mathrm{nd}, 3 \mathrm{rd}, 5 \mathrm{th}, 7 \mathrm{th}$, 9th, and 10th floor levels are used as the observation vector.

The uncertainties of measurement noises on the results of system identification are considered by superimposition of noise process with the theoretically computed response quantities, that is,

$$
\mathbf{y}_{j}=\mathbf{y}_{j 0}+\eta_{j} \nu_{j} \sigma\left(\mathbf{y}_{j 0}\right),
$$

where $\mathbf{y}_{j}$ and $\mathbf{y}_{j 0}$ are the $j$ th set of measured acceleration vector and calculated acceleration vector, respectively, $v_{j}$ is the $j$ th random vector with standard normal random distribution, $\sigma\left(\mathbf{y}_{j 0}\right)$ is the standard deviation of the calculated accelerations $\mathbf{y}_{j 0}$, and $\eta_{j}$ is the level of noise in measurements, which is an important parameter representing the level of uncertainties in the measured accelerations.

Structural damages of the building are assumed as follow: the 3 rd story stiffness $k_{3}$ is reduced by $5 \%$, the 5 th story stiffness $k_{5}$ is reduced by $20 \%$, and the 8 th story stiffness $k_{8}$ is reduced by $10 \%$.

Each set of the measured acceleration responses with uncertainties of measurement noise is used to identify structural physical parameters, structural damage, and the unknown excitation to the building using the deterministic identification algorithm in Section 2. In the probabilistic approach as shown in Section 3, the measured accelerations contaminated by noises are taken as the uncertain variables as shown by (14). The Mote Carlo method is performed with sample size equal to 100 . Two measurement noise levels, $5 \%$ and $20 \%$, are simulated to examine the effectiveness of proposed algorithms.

Figures 1(a)-1(b) show the comparisons of the identification results by the deterministic identification algorithm and the probabilistic approach when the measurement noise levels are equal to $5 \%$ and $20 \%$, respectively. It is seen from Figure 1(a) that the identification values of the relative stiffness change by the deterministic and the probabilistic approach are very close to the true values, indicating that both approaches can identify structural element damage when the measurement noise level is low. However, Figure 1(b) shows that when measurement noise level is quite high, which is equal to $20 \%$, the identification error by the deterministic algorithm increases and the false positives of damages occur in several undamaged floor stiffness, especially the false damage identification of about $7 \%$ reduction of $k_{1}$. On the other hand, the damage index $D_{i}$ in the probabilistic approach can still accurately indicate the location and severity of structural damage as shown in Figure 1(b) and Table 1. This demonstrates that the proposed probabilistic approach can avoid the false identification of damages by the deterministic algorithm.

Figures 2(a)-2(c) compare the identification results of unknown excitation by the deterministic algorithm and the probabilistic approach. Form these comparisons, it is shown that the identification accuracy on the unknown excitation by the deterministic algorithm decreases with the increase of the measurement noise level. There is an obvious deviation between the identified and the true excitations. However, Figure 2(c) demonstrates that the effect of uncertainties of measurement noise on the identified unknown excitation can be diminished by using the statistical average of multisets of identified unknown excitation time histories.

The identification results of structural damage probabilities defined in Section 3.2 for all the elements are summarized in Table 1. It is shown that the damage probabilities of elements 3, 5, and 8 are close to $100 \%$, which are much larger than $50 \%$. The damage probabilities of all the undamaged elements are very close to $50 \%$. This indicates that the proposed identification probability of structural damage can accurately indicate all the structural damages locations.

\section{Conclusions}

In this paper, a probabilistic approach is proposed for the identification of structural damage and unknown external excitations using only limited measurements of structural acceleration responses contaminated by intensive measurement noises. The probabilistic approach is an improvement of the deterministic identification algorithm recently proposed by the authors. Structural parameters and unknown excitation are identified in a sequential manner, which simplifies the identification problem compared with other simultaneous identification algorithms. The statistical parameters of the identified structural parameters are estimated using the statistical theory, and a damage index is defined to indicate the location and severity of structural damage. The probability of identified structural damage is further derived based on the reliability theory. The unknown external excitation on the structure can also be derived by statistical average of multisets of identified unknown excitation timehistories. Therefore, the novelty of the research is that it proposes a probabilistic approach which can accurately identify 


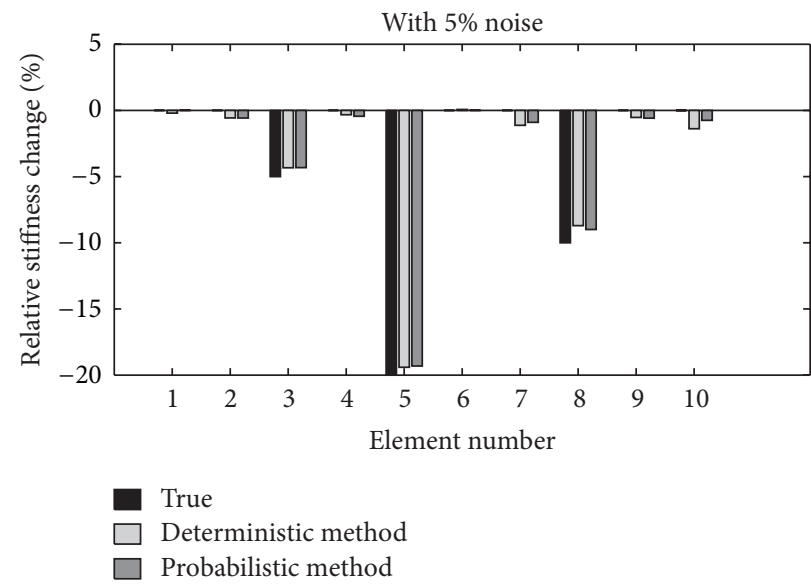

(a) Comparisons of identified stiffness with 5\% measurement noise

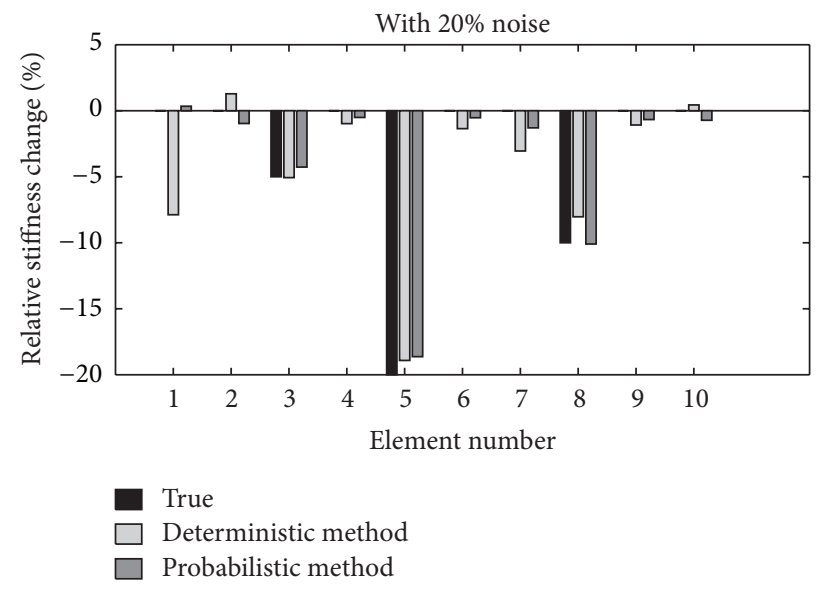

(b) Comparisons of identified stiffness with $20 \%$ measurement noise

FIGURE 1: Comparisons of identification stiffness with different measurement noise levels.

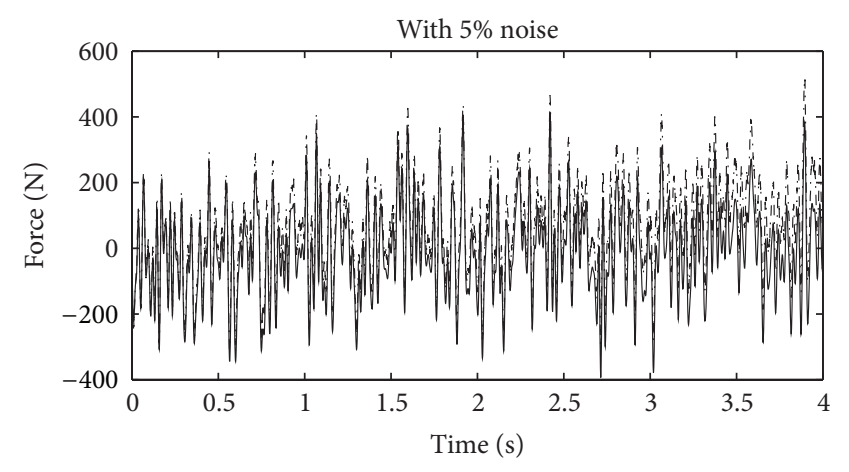

- True

.... Deterministic method

(a) Identified unknown excitation by the deterministic algorithm with $5 \%$ noise

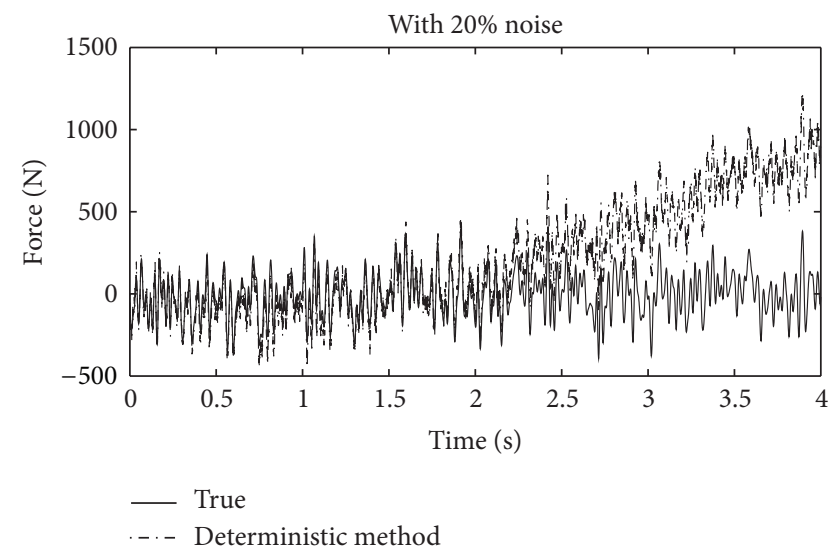

(b) Identified unknown excitation by the deterministic algorithm with $20 \%$ noise

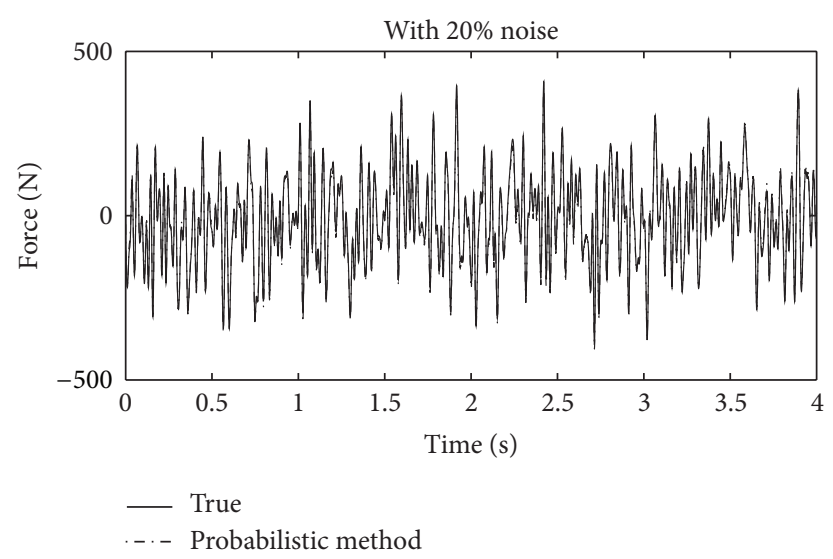

(c) Identified unknown excitation by the probabilistic approach with $20 \%$ noise

FIgURE 2: Comparison of identified unknown excitation. 
TABLE 1: Comparisons of identified damage indices and damage probabilities.

\begin{tabular}{|c|c|c|c|c|c|c|c|}
\hline \multirow{3}{*}{$\begin{array}{l}\text { Story } \\
\text { no. }\end{array}$} & \multirow{3}{*}{ Actual damage (\%) } & \multicolumn{6}{|c|}{ Noise level } \\
\hline & & \multicolumn{3}{|c|}{$5 \%$} & \multicolumn{3}{|c|}{$20 \%$} \\
\hline & & $D_{i}(\%)$ & Error (\%) & $P_{i}(\%)$ & $D_{i}(\%)$ & Error (\%) & $P_{i}(\%)$ \\
\hline 1 & 0.00 & 0.01 & 0.01 & 49.2 & 0.34 & 0.34 & 49.4 \\
\hline 2 & 0.00 & -0.58 & 0.58 & 50.3 & -0.97 & 0.97 & 56.6 \\
\hline 3 & -5.00 & -4.32 & 0.68 & 99.2 & -4.27 & 0.73 & 90.8 \\
\hline 4 & 0.00 & -0.45 & 0.45 & 50.3 & -0.51 & 0.51 & 54.2 \\
\hline 5 & -20.00 & -19.32 & 0.68 & 100 & -18.63 & 1.37 & 100 \\
\hline 6 & 0.00 & 0.01 & 0.01 & 49.9 & -0.53 & 0.53 & 55.7 \\
\hline 7 & 0.00 & -0.900 & 0.900 & 51.8 & -1.30 & 1.30 & 61.4 \\
\hline 8 & -10.00 & -9.00 & 1.00 & 100 & -8.58 & 1.41 & 92.1 \\
\hline 9 & 0.00 & -0.58 & 0.58 & 50.7 & -0.67 & 0.67 & 56.5 \\
\hline 10 & 0.00 & -0.75 & 0.75 & 51.4 & -0.73 & 0.73 & 57.2 \\
\hline Total error & - & - & 0.88 & & - & 0.85 & \\
\hline
\end{tabular}

structural damage and the unknown excitations more than the deterministic identification algorithm under high-level measurement noises. The proposed probabilistic approach is clear and simple compared with other previous algorithms. A numerical simulation example demonstrates that the proposed probabilistic approach can accurately identify structural damage and the unknown excitations using only partial measurements of structural acceleration responses contaminated by intensive measurement noises.

It is important to investigate the efficiency of the proposed probabilistic approach for the identification of other types of structural systems. Moreover, damage identification is only verified by the numerical simulation in this paper. Experimental studies to fully assess the performances of the proposed algorithm are needed. Such work is investigated by the authors and the results will be reported in future.

\section{Acknowledgments}

This research is funded by the National Natural Science Foundation of China (NSFC) through Grant no. 51178406, the research funding SLDRCE10-MB-01 from the State Key Laboratory for Disaster Reduction in Civil Engineering at Tongji University, China, and by the Fujian Natural Science Foundation through Grant no. 2010J01309.

\section{References}

[1] in Proceedings of the 7th and 8th International Workshops on Structural Health Monitoring, F. K. Chang, Ed., Stanford University, Palo Alto, Calif, USA, 2011.

[2] "Structural health monitoring of intelligent infrastructure," in Proceedings of the 4th International Conference on Structural Health Monitoring of Intelligent Infrastructures, U. Meier, B. Havaranek, and M. Motavalli, Eds., Zurich, Switzerland, 2009.

[3] H. Sohn, C. R. Farrar, F. M. Hemez, D. D. Shunk, D. W. Stinemates, and B. R. Nadler, "A review of structural health monitoring literature: 1996-2001," Los Alamos National Laboratory Report LA-13976-MS, 2003.
[4] R. Ghanem and M. Shinozuka, "Structural-system identification. I: theory," Journal of Engineering Mechanics, vol. 121, no. 2, pp. 255-264, 1995.

[5] X. Wang, C. Yang, L. Wang, H. Yang, and Z. Qiu, "Membershipset identification method for structural damage based on measured natural frequencies and static displacements," Structural Health Monitoring, vol. 12, pp. 23-34, 2013.

[6] D. Wang and A. Haldar, "System identification with limited observations and without input," Journal of Engineering Mechanics, vol. 123, no. 5, pp. 504-511, 1997.

[7] M. Katkhuda, R. Martinez, and A. Haldar, "Health assessment at local level with unknown input excitation," Journal of Structural Engineering, vol. 131, no. 6, pp. 956-965, 2005.

[8] L. Jie and C. Jun, "A statistical average algorithm for the dynamic compound inverse problem," Computational Mechanics, vol. 30, no. 2, pp. 88-95, 2003.

[9] J. N. Yang, S. Pan, and S. Lin, "Least-squares estimation with unknown excitations for damage identification of structures," Journal of Engineering Mechanics, vol. 133, no. 1, pp. 12-21, 2007.

[10] M. J. Perry and C. G. Koh, "Output-only structural identification in time domain: numerical and experimental studies," Earthquake Engineering and Structural Dynamics, vol. 37, no. 4, pp. 517-533, 2008.

[11] X. Zhao, Y. L. Xu, J. Li, and J. Chen, "Hybrid identification method for multi-story buildings with unknown ground motion: theory," Journal of Sound and Vibration, vol. 291, no. 12, pp. 215-239, 2006.

[12] J. N. Yang, S. Pan, and H. W. Huang, "An adaptive extended Kalman filter for structural damage identifications II: unknown inputs," Structural Control and Health Monitoring, vol. 14, no. 3, pp. 497-521, 2007.

[13] J. N. Yang and H. W. Huang, "Sequential non-linear leastsquare estimation for damage identification of structures with unknown inputs and unknown outputs," International Journal of Non-Linear Mechanics, vol. 42, no. 5, pp. 789-801, 2007.

[14] B. Xu, J. He, R. Rovekamp, and S. J. Dyke, "Structural parameters and dynamic loading identification from incomplete measurements: approach and validation," Mechanical Systems and Signal Processing, vol. 28, pp. 244-257, 2012. 
[15] Y. Lei, Y. Q. Jiang, and Z. Q. Xu, "Structural damage detection with limited input and output measurement signals," Mechanical Systems and Signal Processing, vol. 28, pp. 229-243, 2012.

[16] Y. Lei and Y. K. Mao, "A technique based on kalman filter and least square estimation for time domain structural damage detection," Advanced Materials Research, vol. 163-167, pp. 26832688, 2011.

[17] P. Kettle, A. Murray, and F. Moynihan, "Sensor less control of a brushless DC motor using an Extended Kalman estimator," in Proceedings of the International Power Conversion Conference, Intelligent Monitoring (PCIM '98), pp. 385-392, 1998.

[18] J. Chen and J. Li, "Simultaneous identification of structural parameters and input time history from output-only measurements," Computational Mechanics, vol. 33, no. 5, pp. 365-374, 2004.

[19] Q. Zhang, L. Jankowski, and Z. Duan, "Simultaneous identification of excitation time histories and parametrized structural damages," Mechanical Systems and Signal Processing, vol. 33, pp. 56-68, 2012.

[20] K.-V. Yuen and L. S. Katafygiotis, "Substructure identification and health monitoring using noisy response measurements only," Computer-Aided Civil and Infrastructure Engineering, vol. 21, no. 4, pp. 280-291, 2006.

[21] X. Y. Li and S. S. Law, "Damage identification of structures including system uncertainties and measurement noise," AIAA Journal, vol. 46, no. 1, pp. 263-276, 2008.

[22] K. Zhang, H. Li, Z. D. Duan, and S. S. Law, "A probabilistic damage identification approach for structures with uncertainties under unknown input," Mechanical Systems and Signal Processing, vol. 25, no. 4, pp. 1126-1145, 2011.

[23] Y. Xia and H. Hao, "Statistical damage identification of structures with frequency changes," Journal of Sound and Vibration, vol. 263, no. 4, pp. 853-870, 2003.

[24] T. Saito, S. Mase, and K. Morita, "A probabilistic approach to structural damage estimation," Structural Control and Health Monitoring, vol. 12, no. 3-4, pp. 283-299, 2005.

[25] M. Chandrashekhar and R. Ganguli, "Uncertainty handling in structural damage detection using fuzzy logic and probabilistic simulation," Mechanical Systems and Signal Processing, vol. 23, no. 2, pp. 384-404, 2009.

[26] R. Palma, G. Rus, and R. Gallego, "Probabilistic inverse problem and system uncertainties for damage detection in piezoelectrics," Mechanics of Materials, vol. 41, no. 9, pp. 10001016, 2009. 


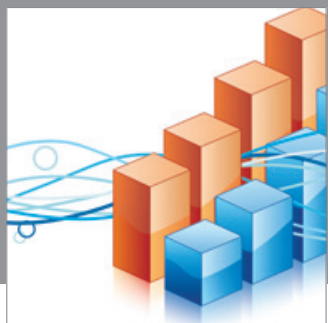

Advances in

Operations Research

mansans

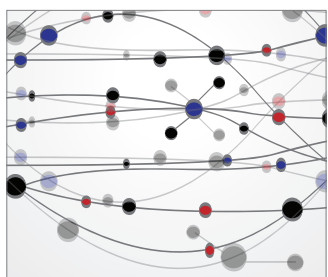

The Scientific World Journal
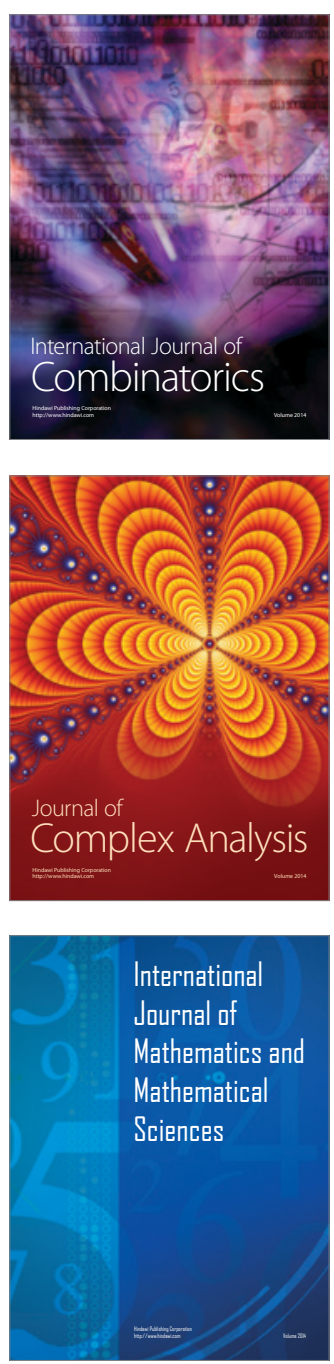
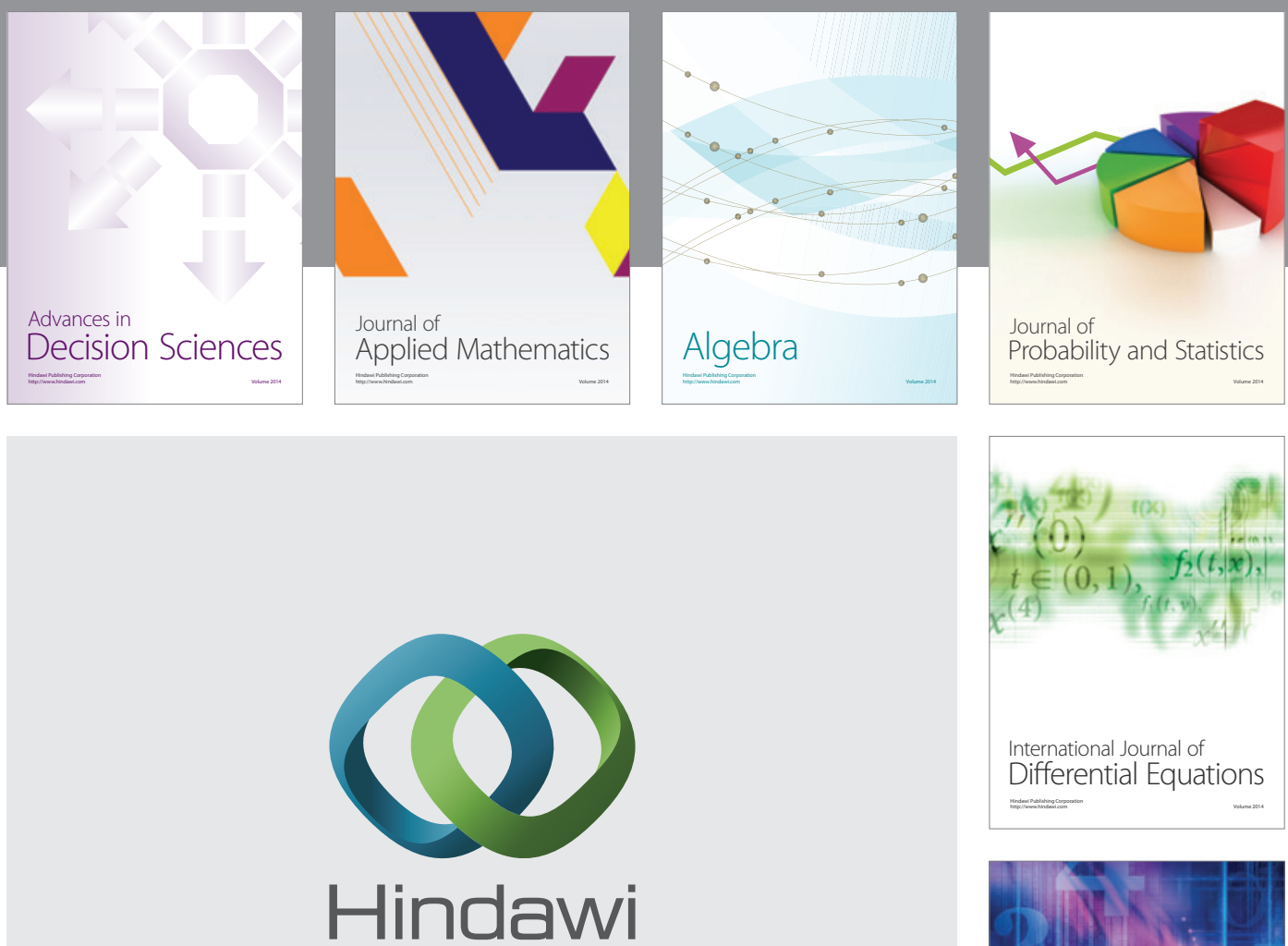

Submit your manuscripts at http://www.hindawi.com
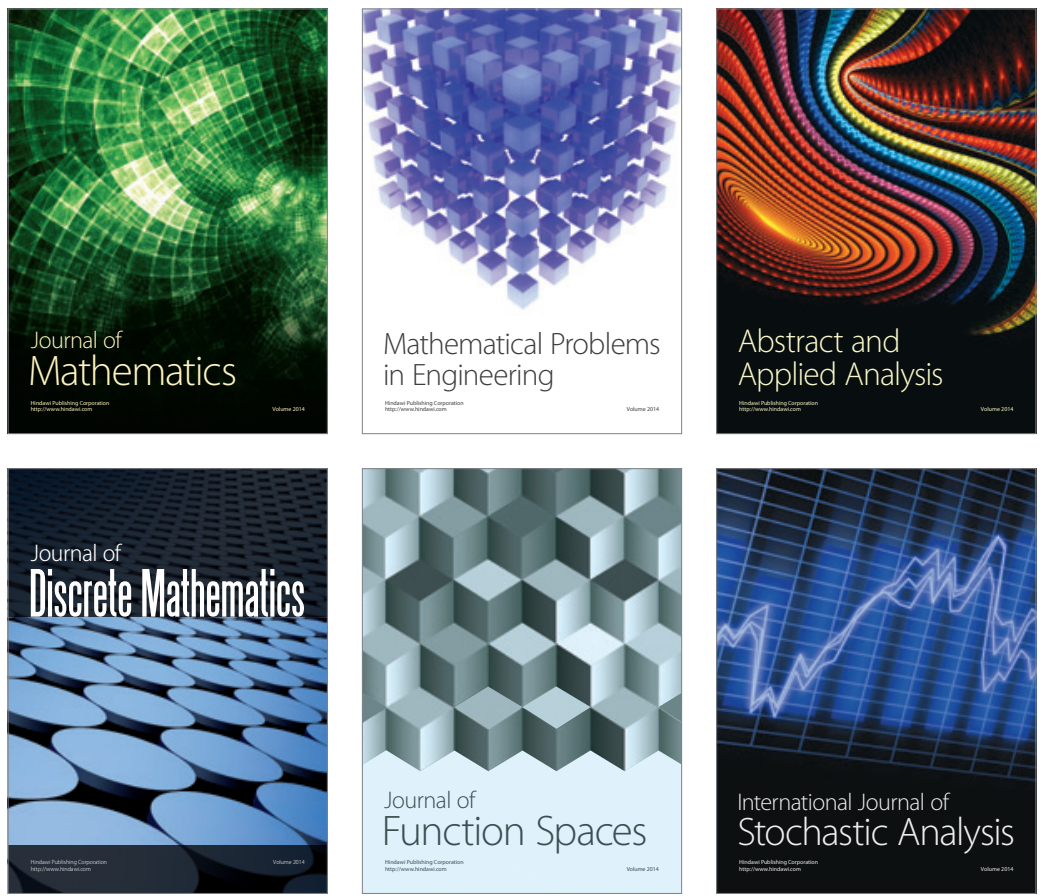

Journal of

Function Spaces

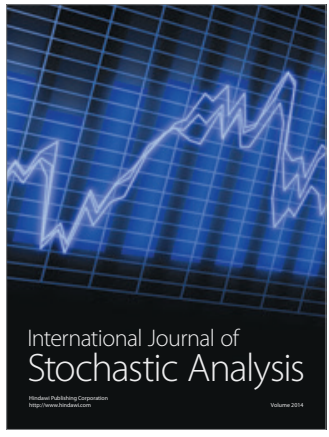

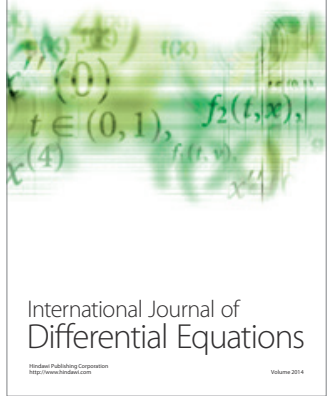
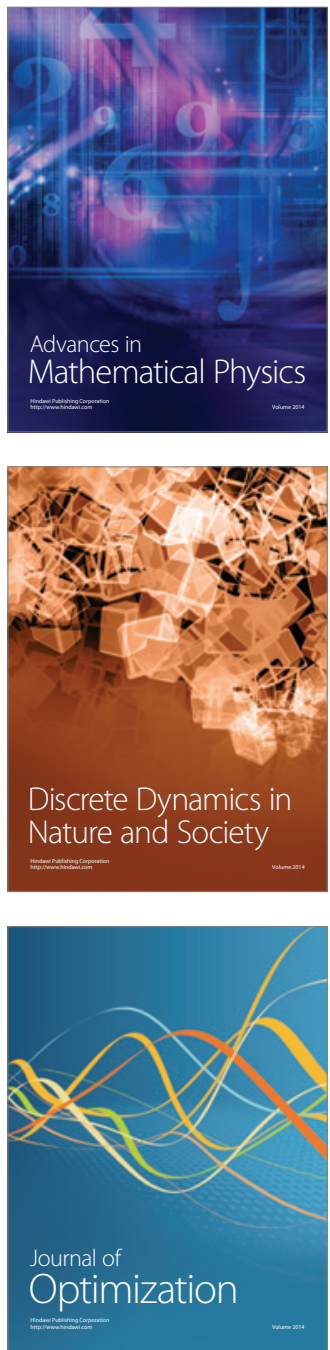